\title{
Taxonomia de Falhas em Programas Concorrentes em Elixir
}

\author{
Matheus Deon Bordignon \\ Universidade Tecnológica Federal do Paraná - UTFPR \\ Dois Vizinhos, PR, Brasil \\ matheusbordignon@alunos.utfpr.edu.br
}

\begin{abstract}
Computer processing capacity is becoming increasingly insufficient and it encourages the use of concurrent programming to develop applications that reduce the computing time. Due to features such as communication, synchronization and non-determinism, concurrent programs may present concurrency-related errors. This paper presents a defect taxonomy for Elixir concurrent programs considering the functions present on Kernel and Task modules. Defect patterns were identified from the insertion of small disturbances into concurrent functions present in a benchmark of concurrent Elixir programs. The association between entered defects and concurrent programming errors has resulted in defect taxonomy for concurrent Elixir programs. The defined taxonomy will be used to support the definition of criteria and testing tools for concurrent Elixir programs.
\end{abstract}

\section{KEYWORDS}

Taxonomia de Falhas, Elixir, Programação Concorrente, Teste de Software

\section{INTRODUÇÃO}

A capacidade de processamento dos computadores mostra-se cada vez mais insuficiente e incentiva o uso de programação concorrente e paralela para desenvolver aplicações que reduzam o tempo computacional. A programação concorrente refere-se ao conceito de construir um programa que contenha no mínimo dois processos (ou threads) que possam ser executados paralelamente e/ou concorrentemente, interagindo na solução de problemas [1]. Enquanto a programação sequencial convencional utiliza primitivas que são executadas sequencialmente, como definições e uso de variáveis, desvios condicionais e incondicionais, estruturas de repetição e chamadas de funções/sub-rotinas/métodos, a programação concorrente procura meios de executar o máximo de tarefas ao mesmo tempo, melhorando o desempenho da aplicação e aumentando a utilização dos recursos computacionais disponíveis [2].

Para criar um software utilizando os conceitos de programação concorrente, três etapas básicas são necessárias: (1) representação do conjunto de tarefas que serão executados concorrentemente, (2) geração e finalização destas tarefas no formato de processos ou threads e (3) coordenação e gerência das interações entre os processos enquanto estes estiverem executando juntos [3].

Para a realização das etapas 2 e 3 , ferramentas de programação concorrente fazem-se necessárias, como linguagens e bibliotecas que suportam a programação concorrente. Essas linguagens e bibliotecas podem ser classificadas de acordo com o paradigma de desenvolvimento a ser utilizado. O paradigma de programação representa o modo como acontece a comunicação e sincronização entre

\author{
Rodolfo Adamshuk Silva \\ Universidade Tecnológica Federal do Paraná - UTFPR \\ Dois Vizinhos, PR, Brasil \\ rodolfoa@utfpr.edu.br
}

os processos. Os dois paradigmas de programação concorrente são a memória compartilhada e a passagem de mensagem.

No paradigma de memória compartilhada, existe um espaço de endereçamento compartilhado, no qual diferentes processos podem fazer operações de leitura e escrita nessas variáveis. Como é possível perceber, será necessário ter um mecanismo de segurança de dados, pois diferentes processos podem acessar a mesma região crítica, por exemplo uma variável que pode ser lida e modificada. Dentre as maneiras de controlar o acesso à região crítica, citam-se os semáforos, barreiras e monitores $[4,5]$.

O paradigma baseado em passagem de mensagem é utilizado para comunicação entre processos que não utilizam um mesmo endereçamento de memória compartilhado. Existem dois elementos base utilizados para a passagem de mensagem: send e receive, que embora possam variar sintaticamente, dependendo da linguagem, tem a mesma semântica. Send compreende no envio de uma mensagem de um processo a outro, enquanto receive é utilizado pelo processo destino para receber a mensagem [5].

Uma linguagem ou biblioteca de programação pode implementar um ou ambos paradigmas de programação. Atualmente, diferentes linguagens emergentes de programação dão suporte à programação concorrente, como Go, Elixir, Ruby, Python entre outras. O Elixir, em especial, foi criada pelo brasileiro José Valim em 2012 e é uma linguagem que suporta o desenvolvimento de aplicações concorrentes e distribuídas de forma dinâmica e moderna. O Elixir, através de uma sintaxe agradável, auxilia o programador a resolver os problemas de concorrência, distribuição e tolerância a falhas semelhante ao Erlang [6].

Um fator que influencia os testes para programas concorrentes é sua natureza não determinística. Programas sequenciais têm característica determinística, isto é, independentemente do número de execuções de um mesmo programa, o mesmo valor de entrada produzirá a mesma saída. Programas concorrentes têm característica não determinística, possibilitando que a execução de um mesmo programa concorrente com o mesmo valor de entrada, produza diferentes resultados. Assim, quando relacionados a programas concorrentes, o teste visa a identificação de erros relacionados à comunicação, ao paralelismo e à sincronização e, portanto, os casos de teste são gerados a partir dessas categorias. Apesar disso, os demais tipos de erro também devem ser considerados e tratados, pois além de sustentar o programa incorreto, ainda podem provocar erros de comunicação, paralelismo e sincronização comentados anteriormente.

A detecção de falhas na execução em Elixir é menos frequente em comparação com as linguagens de programação mais comuns. Os problemas relacionados à sincronização e comunicação (ditos imprevisíveis) são difíceis de reproduzir. Por essa razão, o modo de projetar aplicações chamado "Let it crash" é utilizado nas comunidades Elixir e Erlang. Esse tipo de mentalidade incentiva a 
programação do fluxo principal (conhecido também como caminho feliz ou happy-path), na qual concentra-se primeiro em cenários sem nenhuma condição de erro (estado incorreto no sistema), permitindo concentração no propósito da aplicação e tratar os caminhos de exceção mais tarde (após eles ocorrerem) [7].

Uma forma de aumentar a disponibilidade, confiabilidade de programas em Elixir é refinar possíveis reações a falhas que ocorrem durante o funcionamento do sistema. Em geral, isso pode ser feito por meio de funções que monitoram o funcionamento de processos e reiniciam um processo quando um problema ocorre como, por exemplo, um deadlock. De qualquer maneira, a detecção de falhas e o conhecimento sobre essas falhas são essenciais.

Um dos problemas ao testar programas concorrentes em linguagens emergentes como o Elixir é a falta de estudos sobre as falhas que podem ocorrer pela utilização incorreta das funções de programação concorrente. Por meio de um processo de 5 passos, foi possível definir uma taxonomia de falhas para programas concorrentes em Elixir que utilizam os módulos Kernel e Task.

O artigo está organizado da seguinte forma: Visão geral e as definições são apresentadas na Seção 2. Na Seção 3 são discutidos os trabalhos relacionados. A Seção 4 apresenta a taxonomia de falhas que é seguida por um exemplo de aplicação da taxonomia na Seção 5. Finalmente, as conclusões e perspectivas sobre trabalhos futuros são apresentadas na Seção 6.

\section{VISÃO GERAL E DEFINIÇÕES}

\subsection{Programação concorrente em Elixir}

O Elixir é uma linguagem de programação que segue o paradigma funcional de programação. Herdando do Erlang a capacidade de resolução de problemas de concorrência, distribuição, tolerância a falhas e alta disponibilidade hot swap, a linguagem vem ganhando mais adeptos e vem sendo utilizada em grandes empresas [8].

No Elixir, a comunicação entre processos é feita somente por troca de mensagens, o que permite que a linguagem não tenha que gerenciar o estado (seguro ou não seguro) dessas variáveis [9]. O Elixir possui funções utilizadas para resolver problemas que envolvem paralelismo e concorrência presentes em seis módulos: Kernel, Task, Process, Agent, Genserver e Task.Supervisor. Tabela 1 apresenta as funções dos módulos Kernel e Task, os quais serão explorados na taxonomia. Os números após as funções (como spawn/1) indicam a aridade, ou seja, o número de argumentos que as funções podem receber.

\subsection{Teste de software}

Embora sistemas robustos produzam resultados impressionantes, eles podem trazer enormes problemas para os desenvolvedores. Problemas referentes à sincronização, manipulação e processamento de dados são apenas alguns dos problemas passíveis de acontecer sem o devido tratamento. Para controlar esses problemas, verificando a corretude dos softwares e diminuindo riscos, existe o teste, que pode ser definido como um conjunto de tarefas que podem ser planejadas com antecedência e executadas sistematicamente.

No contexto de teste de software, Engano, Defeito, Erro e Falha são tratados de maneira distinta. Engano refere-se a ação humana que produz um defeito. Defeito é uma definição incorreta de dados, geralmente gerada por engano humano. A existência de um defeito
Table 1: Funções de Programação Concorrente em Elixir nos módulos Kernel e Task

\begin{tabular}{|c|c|}
\hline Função & Descrição \\
\hline $\begin{array}{l}\text { spawn/1 } \\
\text { spawn/3 }\end{array}$ & Criação de processos \\
\hline $\begin{array}{l}\text { spawn_link/1 } \\
\text { spawn_link/3 }\end{array}$ & $\begin{array}{l}\text { Criação de processos com uma ligação } \\
\text { bidirecional }\end{array}$ \\
\hline $\begin{array}{l}\text { spawn_monitor/1 } \\
\text { spawn_monitor } / 3\end{array}$ & $\begin{array}{l}\text { Criação de processos com uma ligação } \\
\text { unidirecional }\end{array}$ \\
\hline $\begin{array}{l}\text { send } / 2 \\
\text { receive } / 1\end{array}$ & $\begin{array}{l}\text { Envio de mensagem } \\
\text { Recebimento de mensagens }\end{array}$ \\
\hline $\begin{array}{l}\text { Task.async/1 } \\
\text { Task.async/3 }\end{array}$ & Inicia uma tarefa que deve ser aguardada \\
\hline $\begin{array}{l}\text { Task.async_stream/3 } \\
\text { Task.async_stream/5 }\end{array}$ & $\begin{array}{l}\text { Executa a função concorrentemente em cada } \\
\text { valor do enumerable }\end{array}$ \\
\hline Task.await/2 & Aguarda uma resposta da tarefa e a retorna \\
\hline Task.child_spec/1 & $\begin{array}{l}\text { Retorna uma especificação para iniciar uma } \\
\text { tarefa sob um supervisor }\end{array}$ \\
\hline Task.shutdown/2 & $\begin{array}{l}\text { Desvincula e desliga a tarefa e, em seguida, } \\
\text { procura uma resposta }\end{array}$ \\
\hline $\begin{array}{l}\text { Task.start/1 } \\
\text { Task.start/3 }\end{array}$ & Inicia uma tarefa \\
\hline $\begin{array}{l}\text { Task.start_link/1 } \\
\text { Task.start_link/3 }\end{array}$ & Inicia um processo vinculado ao processo atual \\
\hline $\begin{array}{l}\text { Task.yield/2 } \\
\text { Task.yield_many/2 }\end{array}$ & $\begin{array}{l}\text { Bloqueia temporariamente o processo atual, } \\
\text { aguardando a resposta da tarefa }\end{array}$ \\
\hline
\end{tabular}

pode gerar um erro durante a execução do programa, ou seja, um estado inconsistente ou ação inesperada do programa. Este erro pode ocasionar uma falha que é o resultado diferente do resultado esperado [2]. O termo defeito será utilizando para problemas sintáticos cometidos pelos desenvolvedores. Já os termos erro e falha serão utilizados para o resultado final que pode ser observado ou inferenciado (por exemplo deadlock que não é uma resposta do sistema, mas sim o estado em que ele se encontra).

\section{TRABALHOS RELACIONADOS}

Para a definição de técnicas e critérios de teste no contexto de programas concorrentes, dois pontos importantes devem ser considerados: 1) os tipos de erros que devem ser evidenciados pelos critérios de teste; e 2) como representar o programa concorrente de modo a obter as informações necessárias para os critérios de teste [2]. No contexto de sistemas computacionais tolerantes a falhas, a técnica de teste baseada em defeitos é aplicada por meio do critério de injeção de falhas [10]. Para que esse critério seja aplicado e visando atender ao primeiro ponto importante no teste de programas concorrente, uma taxonomia de falhas faz-se necessária.

Diferentes taxonomias de defeitos/erro/falha foram definidas na literatura para linguagens de programação como Ada [11], MPI [1216], C e C ++ [17] e SystemC [18]. Farchi et al. [19] apresentam uma taxonomia de defeitos para programas Java multithreading. Embora a taxonomia tenha sido desenvolvida para a linguagem Java, ela pode ser aplicada em outros contextos independente da linguagem de programação. Os defeitos são categorizados com relação a (1) Código desprotegido: (a) Operação não atômica dada como atômica, (b) Acesso em dois estágios, (c) Lock errado ou inexistente e (d) Bloqueio de dupla checagem; (2) Interleavings: (a) Uso de sleep para 
sincronização e (b) Perda de notify; e (3) Bloqueio ou morte de thread: (a) Bloqueio em região crítica e (b) Thread órfã.

Levando em consideração os trabalhos de Vetter e Supinski [12], Krammer et al. [14] e Luecke et al. [13], DeSouza et al. [15] apresentam uma taxonomia de erros para programas desenvolvidos em MPI. A taxonomia apresentada divide os erros em três categorias principais: (1) Sincronização subdividido em (a) Deadlock: Padrão e Dependente do tempo e (b) Condição de corrida: Interface e Entre processos; (2) Incompatibilidade, subdividido em (a) Tipo de chamada, (b) Argumentos e (c) Tamanho; e (3) Recursos, subdividido em (a) Alocação, (b) Inicialização e (c) Desalocação.

Lopez et al. [20] apresentam uma taxonomia de erros de concorrência em programas baseados em atores. A taxonomia leva em consideração programas desenvolvidos em Erlang, Actor-Foundry, Scala e Java Script. A taxonomia é composta por duas categorias. A primeira é denominada Falta de Progresso, na qual estão os erros de (1) Deadlock de comunicação, (2) Deadlock comportamental e (3) Livelock. A segunda categoria é denominada Violação de Protocolo de Mensagem na qual estão os erros de (1) Violação da ordem de mensagem, (2) Combinação incorreta de mensagem e (3) Inconsistência de memória. Como validação da taxonomia, um catálogo de defeitos concorrentes coletados da literatura foi classificado segundo a taxonomia definida.

\section{TAXONOMIA DE FALHAS}

Com base nos artigos apresentados na Seção 3, não há uma definição de processo para a definição de uma taxonomia de falhas/defeitos. Porém, ao observar os trabalhos relacionados, as abordagens utilizadas podem ser divididas em duas categorias: estática e dinâmica.

$\mathrm{Na}$ categoria estática estão as abordagens que realizam um estudo preliminar na linguagem e nos possíveis defeitos que o programador pode inserir nos programas concorrentes e como esses defeitos podem ocasionar falhas. Nessa categoria, os possíveis defeitos são obtidos a partir do conhecimento dos autores da abordagem em relação à linguagem de programação e como pequenas mudanças sintáticas podem alterar a semântica da aplicação.

Na categoria dinâmica estão os trabalhos em que um estudo é feito levando em consideração a análise de código com defeito desenvolvido por programadores. Nessa categoria estão trabalhos em que os autores aplicam um experimento para coletar informações de defeitos reais inseridos por programadores ao desenvolver aplicações concorrentes. Com base na análise de defeitos nos códigos, a taxonomia de defeitos/falhas é desenvolvida.

Este trabalho está inserido na categoria estática de definição de taxonomia de defeitos, na qual somente a sintaxe da linguagem de programação é considerada e a taxonomia de falha é gerada a partir da análise dos autores sobre as funções e os possíveis enganos que podem ser cometidos pelos programadores ao desenvolver aplicações concorrentes em Elixir. Cada defeito do conjunto de defeitos definidos foi semeado em códigos e executou-se para que as falhas pudessem ser obtidas.

O trabalho de Lopez et al. [20] é o mais próximo da taxonomia proposta neste artigo, uma vez que programas na linguagem Erlang foram utilizados no processo de definição da taxonomia. O Elixir é executado pela máquina virtual do Erlang e possui características semelhantes ao Erlang. Desta forma, algumas categorias de falhas foram extraídas dessa taxonomia. Porém, como a taxonomia proposta neste artigo é somente para Elixir, não foi possível utilizar todas as categorias da taxonomia de Lopez et al. [20] e houve a necessidade de adicionar novas categorias.

Para a definição da taxonomia de falhas para programas concorrentes em Elixir, o processo mostrado na Figura 1 foi seguida. $\mathrm{O}$ processo divide-se em 4 etapas:

(1) Identificação das funções concorrentes

(2) Agrupamento de funções com semântica semelhante

(3) Definição de defeitos

(4) Execução e coleta de falhas

Cada uma das etapas é apresenta com mais detalhes nas subseções a seguir.

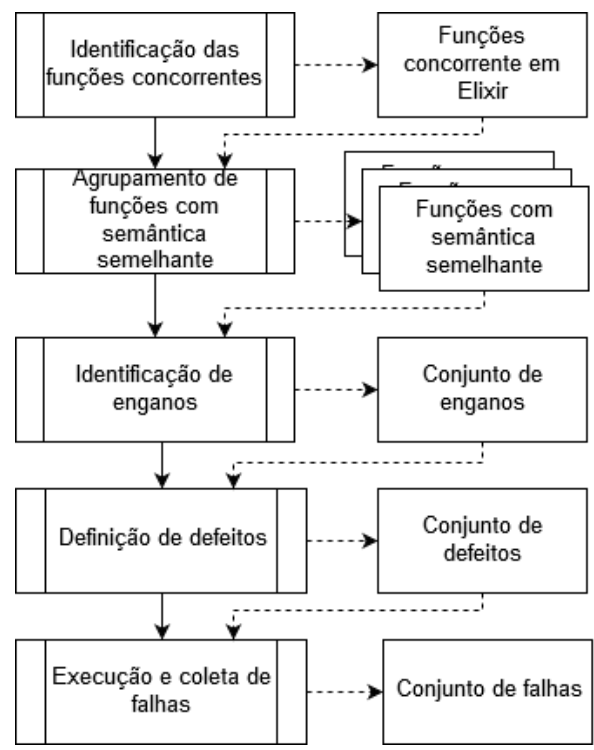

Figure 1: Processo de definição da taxonomia de falhas para funções concorrentes em Elixir

\subsection{Identificação da funções concorrentes}

A partir da análise da documentação da linguagem de programação Elixir ${ }^{1}$, identificou-se os módulos que apresentam funções de programação concorrente disponíveis na linguagem. Como resultado, encontrou-se que funções que permitem a programação concorrente estão distribuídas nos módulos: Kernel, Task, Process, Agent, Genserver e Task.Supervisor. Este trabalho considera apenas as funções do Kernel e no módulo Task (Tabela 1) por serem os mais utilizados e difundidos pela comunidade de desenvolvedores.

\subsection{Agrupamento de funções com semântica semelhante}

O segundo passo consistiu no agrupamento de funções com semântica semelhante. Duas funções são consideradas de semântica semelhante quando possuem objetivos similares no contexto da programação, diferenciando-se entre si, por exemplo, em como uma ação

\footnotetext{
${ }^{1}$ https://elixir-lang.org/docs.html
} 
é realizada pelo programa. O objetivo desse passo é identificar funções que possuem o mesmo objetivo, porém que se diferem entre si por alguma característica especial.

A Tabela 2 apresenta o agrupamento das funções. Por exemplo, um conjunto de funções agrupadas no módulo Kernel é composto por funções de criação de processos spawn/1, spawn/3, spawn_link/1, spawn_link/3, spawn_monitor/1 e spawn_monitor/3. Essas funções estão no mesmo grupo uma vez que elas realizam a criação de processos e são semelhantes semanticamente. Porém, sintaticamente elas diferem-se primeiramente pela aridade, sendo que uma recebe um parâmetro e a outra recebe três parâmetros. Além disso, as funções spawn, spawn_link e spawn_monitor diferem-se entre si pois a função spawn_link mantém um vínculo de ligação com o processo pai (que cria o processo) e a função spawn_monitor faz com que o processo criado seja monitorado pelo processo pai.

Table 2: Agrupamento de funções com semântica semelhante nos módulos Kernel e Task

\begin{tabular}{|c|c|}
\hline Grupo & Função \\
\hline Funções de Criação de Processos no Kernel & $\begin{array}{l}\text { spawn / } 1 \\
\text { spawn / } 3 \\
\text { spawn_link / } 1 \\
\text { spawn_link / } 3 \\
\text { spawn_monitor / } 1 \\
\text { spawn_monitor / } 3\end{array}$ \\
\hline Função de Envio de Mensagem no Kernel & send / 2 \\
\hline $\begin{array}{l}\text { Função de Recebimento de Mensagem no } \\
\text { Kernel }\end{array}$ & receive \\
\hline Funçõe de criação de tarefas no módulo Task & $\begin{array}{l}\text { Task.start / } 1 \\
\text { Task.start / } 3 \\
\text { Task.start_link / } 1 \\
\text { Task.start_link / } 3 \\
\text { Task.async / } 1 \\
\text { Task.async / } 3 \\
\text { Task.async_stream / } 3 \\
\text { Task.async_stream / } 5\end{array}$ \\
\hline $\begin{array}{l}\text { Funções de respostas de tarefas no módulo } \\
\text { Task }\end{array}$ & $\begin{array}{l}\text { Task.await / } 1 \\
\text { Task.yield / } 2 \\
\text { Task.yield_many / } 2\end{array}$ \\
\hline $\begin{array}{l}\text { Função de encerramento de tarefas no mó- } \\
\text { dulo Task }\end{array}$ & Task.shutdown / 2 \\
\hline $\begin{array}{l}\text { Função de monitoramento e supervisão de } \\
\text { tarefas no módulo Task }\end{array}$ & Task.child_spec / 1 \\
\hline
\end{tabular}

\subsection{Definição de enganos}

Após a definição dos grupos de funções com semântica semelhantes, realizou-se uma análise sintática das funções de cada grupo, observando os parâmetros de cada função e destacando as mudanças sintáticas entre elas. Por exemplo, as funções de spawn/1, spawn_link/1 e spawn_monitor/1 diferem-se entre si sintaticamente por possuir um sufixo _link, _monitor ou por não possuir sufixo como visto a seguir:

spawn(fn -> do_something() end)

spawn_link(fn -> do_something() end)

spawn_monitor(fn -> do_something() end)
A partir dessa análise, identificou-se possíveis enganos que os programadores podem cometer ao utilizar cada grupo de função que possuem sintaxe semelhante e semântica diferente, porém parecida, como é o caso das funções de criação de processos spawn.

Como resultado, obteve-se um conjunto de possíveis enganos que um programador pode cometer ao programar utilizando as funções concorrentes em Elixir dos módulos Kernel e Task. Esse conjunto de enganos serve como base para a definição da taxonomia de defeitos mostrada na próxima seção.

\subsection{Definição de defeitos}

A taxonomia de defeitos apresentada abaixo simula os enganos que podem ser cometidos por programadores durante o desenvolvimento de programas concorrentes em Elixir utilizando as funções presentes nos módulos Kernel e Task.

Cinco categorias de defeitos foram definidas: (1) Defeitos por troca de função, (2) Defeitos por ausência de função, (3) Defeitos por ausência de parâmetros, (4) Defeitos por adição de parâmetro e (5) Defeitos por troca de parâmetro. Os defeitos por troca de função correspondem às alterações nas chamadas das funções. Os defeitos por ausência de função simulam esquecimentos cometidos por programadores na chamada de funções. As diferentes aridades de algumas funções do Elixir podem levar a enganos durante o desenvolvimento de software. Os defeitos causados pela ausência ou por adição de parâmetros entram nesse contexto. Os defeitos por troca de parâmetros estão relacionados ao uso da palavra-chave __MODULE__ que pode ser utilizada quando a função a ser executada está no módulo atual de execução.

Os defeitos identificados para o módulo Kernel podem ser observados na Tabela 3 e os defeitos identificados para o módulo Task podem ser observados na Tabela 4. A primeira coluna da tabela representa a categoria dos defeitos. A segunda coluna apresenta a função alvo na qual deverá ser inserido o defeito e a última coluna apresenta a função com o defeito inserido. Por exemplo, a primeira linha da Tabela 3 apresenta o defeito da Troca de função spawn/1 por spawn_link/1.

Essa taxonomia de defeitos é utilizada para semear defeitos em programas para que a taxonomia de falhas seja definida, como mostrado na próxima seção.

\subsection{Execução e coleta de falhas}

O último passo consistiu na semeadura de defeitos em código concorrente Elixir, a execução desses códigos e a coleta das falhas encontradas. Nesse passo, buscou-se simular as possíveis falhas que podem ser causadas pela inserção dos defeitos definidos no passo anterior. Como resultado, foram obtidas falhas que foram agrupadas em Exceções da linguagem Elixir e Falhas relacionadas à programação concorrente. As Tabelas 5 e 6 apresentam as falhas obtidas no Kernel e módulo Task respectivamente.

O grupo Exceções da linguagem Elixir contém três falhas: (1) Argument Error, (2) Function Clause Error e, (3) Match Error. Tais falhas são lançadas pela própria máquina virtual do Erlang na qual os processos em Elixir são executados.

(1) Argument Error: Esta falha é obtida durante a utilização de funções com parâmetros incorretos. Um exemplo é a função spawn que espera como parâmetro a função que será executa. 
Table 3: Categoria de defeitos do módulo Kernel

\begin{tabular}{|c|c|c|}
\hline Categoria & Função & Defeito \\
\hline \multirow{12}{*}{ Troca de função } & spawn/1 & spawn_link/1 \\
\hline & spawn/1 & spawn_monitor/1 \\
\hline & spawn/3 & spawn_link/3 \\
\hline & spawn/3 & spawn_monitor $/ 3$ \\
\hline & spawn_link/1 & spawn/1 \\
\hline & spawn_link/1 & spawn_monitor/1 \\
\hline & spawn_link/3 & spawn/3 \\
\hline & spawn_link/3 & spawn_monitor $/ 3$ \\
\hline & spawn_monitor/1 & spawn/1 \\
\hline & spawn_monitor/1 & spawn_link/1 \\
\hline & spawn_monitor/3 & spawn/3 \\
\hline & spawn_monitor/3 & spawn_link/3 \\
\hline \multirow{9}{*}{ Deleção de função } & spawn/1 & $/ /$ \\
\hline & spawn/3 & // \\
\hline & spawn_link/1 & $/ /$ \\
\hline & spawn_link/3 & $/ /$ \\
\hline & spawn_monitor/1 & // \\
\hline & spawn_monitor/3 & $/ /$ \\
\hline & self $/ 0$ & // \\
\hline & send $/ 2$ & $/ /$ \\
\hline & receive/1 & // \\
\hline \multirow{5}{*}{ Deleção de parâmetro } & spawn/3 & spawn/1 \\
\hline & spawn_link/3 & spawn_link/1 \\
\hline & spawn_monitor/3 & spawn_monitor/1 \\
\hline & Receive/2 & Receive/1 \\
\hline & Receive/2 & Receive/1 \\
\hline Adição de parâmetro & Receive/1 & Receive/2 \\
\hline \multirow{3}{*}{ Troca de parâmetro } & Spawn/3 & __MODULE \\
\hline & Spawn_link/3 & __MODULE_ \\
\hline & Spawn_monitor $/ 3$ & __MODULE \\
\hline
\end{tabular}

Caso seja passado um número, uma string ou qualquer outro dado que não seja uma função, será obtido Argument Error.

(2) Function Clause Error: Esta falha é lançada quando uma função é executada com parâmetros que não a possibilitam ser executada. Embora seu conceito seja muito semelhante ao Argument Error, a diferença é que no Argument Error a função tenta executar seu objetivo com o valor recebido como parâmetro e dispara o erro quando o processo não é concluído. Function Clause Error acontece quando a função verifica os parâmetros recebidos e em caso de dados incorretos, nem inicia seu processo. Um erro de Function Clause Error pode-se obtido ao chamar a função Task.yield_many passando como parâmetro somente uma tarefa, sendo que a mesma espera uma lista de tarefas como parâmetro.

(3) Match Error: Esta falha acontece na tentativa de corresponder um dado a uma variável sendo que ambos não são compatíveis. Esta falha pode ser obtida, por exemplo, ao atrelar a uma tupla de tamanho dois o retorno da função spawn, já que está retorna somente um valor. Por exemplo, na linha de código: $\{$ pid,reff $=\operatorname{spawn}(f n->\operatorname{task}()$ end $)\}$, o retorno da função spawn é o identificador do processo criado. No exemplo, caso este valor fosse atrelado a uma tupla de tamanho dois, um Match Error seria obtido.
Table 4: Categoria de defeitos do módulo Task

\begin{tabular}{|c|c|c|}
\hline Categoria & Função & Defeito \\
\hline \multirow{18}{*}{ Troca de Função } & async/1 & start/1 \\
\hline & async/1 & start_link/1 \\
\hline & async/3 & start/3 \\
\hline & async/3 & start_link/3 \\
\hline & start/1 & start_link/1 \\
\hline & start/1 & async/1 \\
\hline & start $/ 3$ & start_link/3 \\
\hline & start $/ 3$ & async/3 \\
\hline & start_link/1 & start/1 \\
\hline & start_link/1 & async/1 \\
\hline & start_link/3 & start/3 \\
\hline & start_link/3 & async/3 \\
\hline & await/2 & yield $/ 2$ \\
\hline & await/2 & yield_many/2 \\
\hline & yield/2 & await/2 \\
\hline & yield/2 & yield_many/2 \\
\hline & yield_many/2 & await/2 \\
\hline & yield_many/2 & yield/2 \\
\hline \multirow{13}{*}{ Deleção de Funções } & async/1 & // \\
\hline & async/3 & // \\
\hline & async_stream $/ 3$ & // \\
\hline & async_stream/5 & // \\
\hline & await/2 & // \\
\hline & child_spec/1 & // \\
\hline & shutdown/2 & // \\
\hline & start/1 & // \\
\hline & start/3 & $/ /$ \\
\hline & start_link/1 & // \\
\hline & start_link/3 & $/ /$ \\
\hline & yield/2 & // \\
\hline & yield_many/2 & // \\
\hline \multirow{7}{*}{ Deleção de Parâmetros } & async/3 & async/1 \\
\hline & async_stream $/ 5$ & async_stream $/ 3$ \\
\hline & start/3 & start/1 \\
\hline & start_link/3 & start_link/1 \\
\hline & await/2 & await/1 \\
\hline & yield/2 & yield/1 \\
\hline & yield_many/2 & yield_many/1 \\
\hline \multirow{3}{*}{ Troca de Argumento } & Async/3 & __MODULE_ \\
\hline & Start $/ 3$ & _MODULE \\
\hline & Start_link/3 & _ MODULE__ \\
\hline
\end{tabular}

Table 5: Falhas do módulo Kernel para cada categoria de defeitos

\begin{tabular}{ll}
\hline Categoria & Falha \\
\hline \multirow{2}{*}{ Troca de função } & $\begin{array}{l}\text { Finalização precoce } \\
\text { Deadlock comportamental } \\
\text { Match Error }\end{array}$ \\
\hline Deleção de função & $\begin{array}{l}\text { Finalização Precoce } \\
\text { Deadlock comportamental }\end{array}$ \\
\hline Deleção de parâmetro & $\begin{array}{l}\text { Argument Error } \\
\text { Deadlock comportamental }\end{array}$ \\
\hline Adição de parâmetro & Finalização precoce \\
\hline Troca de parâmetro & Dados incorretos \\
\hline
\end{tabular}

O grupo Falhas relacionadas à programação concorrente contém falhas relacionadas ao comportamento do programa e resultado 
Table 6: Falhas do módulo Task para cada categoria de defeitos

\begin{tabular}{ll}
\hline Categoria & Falha \\
\hline \multirow{3}{*}{ Troca de Função } & Function Clause Error \\
& Finalização precoce \\
& Argument Error \\
& Deadlock comportamental \\
& Dados incorretos \\
\hline \multirow{3}{*}{ Deleção de Funções } & Function Clause Error \\
& Intercalação de mensagem incorreta \\
& Finalização precoce \\
& Dados incorretos \\
\hline \multirow{3}{*}{ Deleção de Parâmetros } & Function Clause Error \\
& Finalização precoce \\
& Intercalação de mensagem incorreta \\
\hline Troca de parâmetro & Dados incorretos \\
\hline
\end{tabular}

gerado. Nesta categoria estão presentes: (1) Dados incorretos, (2) Deadlock comportamental, (3) Finalização precoce e (4) Intercalação de mensagem incorreta.

(1) Dados Incorretos: Esta falha está associada ao resultado incorreto produzido pelo programa. Um exemplo pode ser simulado com a substituição do primeiro parâmetro da função spawn pela tag _ MODULE__ em um programa de calculadora com diversos módulos para realizar cada operação. Caso a chamada da função spawn para o cálculo da soma seja alterada conforme comentado, pode ser obtido qualquer valor diferente da soma esperada.

(2) Deadlock Comportamental: Este erro está relacionado ao comportamento do sistema que encontra-se travado. O problema acontece durante a troca de mensagens entre processos, no qual um processo fica esperando uma mensagem que nunca lhe será enviada. Um exemplo de Deadlock comportamental pode ser gerado ao realizar a substituição da função spawn_link por spawn e induzir um erro de tipos incorretos de dados no processo. Nesta simulação, spawn_link iria encerrar ambos os processos ao lançar a exceção de tipo de dados, enquanto com spawn, o processo filho lançaria a exceção e o processo pai ficaria travado esperando a resposta

(3) Finalização Precoce: Erros de finalização precoce ocorrem quando o programa termina a execução sem ter terminado por completo o seu processamento. Por exemplo, ao utilizar a função spawn para a criação de processo, o processo criador não é finalizado caso o processo criado tenha algum problema. Isso não ocorre quando a função spawn_link é utilizada. Ao trocar essas funções, o processo criador é finalizado antes de completar a sua execução. Outro exemplo do erro é a deleção de uma função spawn que continha um retorno assíncrono, já que ao executar o programa sem a função, nada é executado e o programa finaliza sua execução.

(4) Intercalação de Mensagem Incorreta Esta falha acontece quando o resultado produzido pelo programa difere do esperado. Por exemplo, um programa deve retornar o valor da soma de dois números seguido da multiplicação entre eles, porém retorna primeiro a multiplicação e depois a soma. O problema está associado a funções de recebimento de mensagens, como por exemplo a substituição da função Task.await por Task.yield, já que enquanto a primeira aguarda a mensagem, Task.yield somente verifica se a mensagem chegou e não fica bloqueada.

A Figura 2 apresenta as categorias da taxonomia de falhas e os defeitos relacionados a elas.

\section{APLICAÇÃO DA TAXONOMIA DE FALHAS}

Nesta seção, é apresentada uma aplicação da taxonomia de falhas definida para programas concorrentes em Elixir a qual engloba as funções dos módulos Kernel e Task. O objetivo desse exemplo é mostrar como a taxonomia de falhas pode ser utilizada como suporte ao teste de programas concorrentes em Elixir. Para isso, os seguintes passos foram realizados: (1) Seleção do conjunto de programas, (2) Inserção manual dos defeitos, (3) Execução dos programas defeituosos e (4) Coleta das falhas.

Para a aplicação da taxonomia de falhas em programas concorrentes em Elixir, um benchmark de programas concorrentes em Elixir foi utilizado como base para a semeadura dos defeitos e coleta das falhas. O benchmark utilizado foi definido em Bordignon e Silva [21] e representa as funções do Kernel e do módulo Task em Elixir (Tabela 7). O benchmark é composto por 11 programas de diferentes tamanhos (medidos por LoC) nos quais busca-se explorar as diferentes aridades de algumas funções, como a spawn, spawn_monitor e spawn_link.

\section{Table 7: Programas concorrentes em Elixir [21]}

\begin{tabular}{l|l|l|l}
\hline Id & Nome do Arquivo & Descrição do Problema & LoC \\
\hline P01 & Exemplo Spawn_Monitor & $\begin{array}{l}\text { Maior valor e monitor de } \\
\text { tarefas }\end{array}$ & 15 \\
\hline P02 & Yield_many & Gerenciador de Tarefas & 21 \\
\hline P03 & Elixir Study & $\begin{array}{l}\text { Tarefa com operação de } \\
\text { soma }\end{array}$ & 22 \\
\hline P04 & Events & Vida de um processo & 26 \\
\hline P05 & Agents and Tasks in Elixir & $\begin{array}{l}\text { Execução de tarefas sem re- } \\
\text { sposta }\end{array}$ & 28 \\
\hline P06 & Synchronous Task Stream & Fluxo de Tarefas Síncronas & 55 \\
\hline P07 & Pangram & Pangrama & 56 \\
\hline P08 & Parallel Letter Frequency & Frequência Letra Paralelo & 76 \\
\hline P09 & 17-dining-philosophers & Jantar dos Filósofos & 100 \\
\hline P10 & Parallel Letter Frequency & Frequência Letra Paralelo & 184 \\
\hline P11 & Elixir-sorting & $\begin{array}{l}\text { QuickSort e MergeSort } \\
\text { Paralelo }\end{array}$ & 481 \\
\hline
\end{tabular}

Para cada programa presente no benchmark (Tabela 7), foi realizada uma busca manual por cada função de concorrência e semeado um defeito para cada ocorrência da função na taxonomia de defeitos (Tabela 3 e Tabela 4). Como resultado, um conjunto de programas defeituosos foi gerado, como mostram a Tabela 8 e a Tabela 9. Nessas tabelas, cada linha apresenta o número de programas defeituosos gerados para cada uma das categorias da taxonomia de defeitos.

Para a execução dos programas, um conjunto de dados de teste foi selecionado observando as condições de Alcançabilidade, Infecção e Propagação (R.I.P - Reachability, Infection and Propagation) que o dado de teste possuía com relação ao defeito semeado. Como resultado, foi definido um conjunto de casos de teste que é adequado 


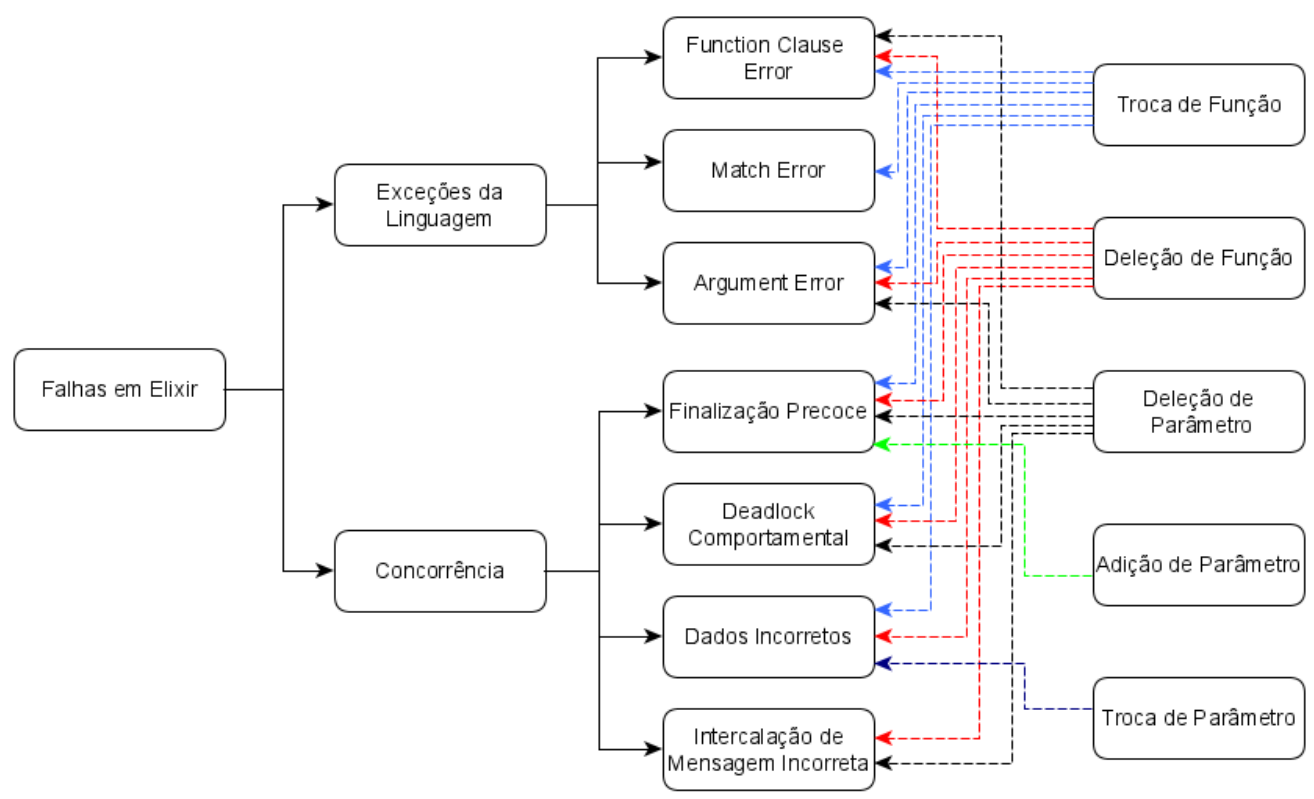

Figure 2: Visão geral das falhas obtidas

Table 8: Defeitos semeados nas funções do módulo Kernel

\begin{tabular}{|c|c|c|c|c|c|c|}
\hline \multirow{2}{*}{ Programa } & \multicolumn{5}{|c|}{ Categoria } & \multirow{2}{*}{ Total } \\
\hline & Troca de função & Deleção de função & Deleção de parâmetro & Adição de parâmetro & Troca de parâmetro & \\
\hline Exemplo Spawn_Monitor & 1 & 1 & 0 & 0 & 0 & 2 \\
\hline Yield_Many & 0 & 0 & 0 & 0 & 0 & 0 \\
\hline Elixir Study & 0 & 0 & 0 & 0 & 0 & 0 \\
\hline Events & 0 & 3 & 2 & 2 & 0 & 7 \\
\hline Agents and Tasks in Elixir & 0 & 0 & 0 & 0 & 0 & 0 \\
\hline Synchronous Task Stream & 0 & 0 & 0 & 0 & 0 & 0 \\
\hline Pangram & 0 & 0 & 0 & 0 & 0 & 0 \\
\hline Parallel Letter Frequency & 0 & 0 & 0 & 0 & 0 & 0 \\
\hline 17-dining-philosophers & 6 & 11 & 4 & 3 & 6 & 30 \\
\hline Parallel Letter Frequency & 1 & 6 & 3 & 2 & 0 & 12 \\
\hline Elixir Sorting & 4 & 6 & 2 & 2 & 0 & 14 \\
\hline Total & 14 & 29 & 11 & 9 & 6 & 65 \\
\hline
\end{tabular}

Table 9: Defeitos semeados nas funções do módulo Task

\begin{tabular}{|c|c|c|c|c|c|c|}
\hline \multirow{2}{*}{ Programa } & \multicolumn{5}{|c|}{ Categoria } & \multirow{2}{*}{ Total } \\
\hline & Troca de função & Deleção de função & Deleção de parâmetro & Adição de parâmetro & Troca de parâmetro & \\
\hline Exemplo Spawn_Monitor & 1 & 0 & 0 & 0 & 0 & 1 \\
\hline Yield_Many & 0 & 1 & 1 & 0 & 0 & 2 \\
\hline Elixir Study & 2 & 2 & 0 & 0 & 0 & 4 \\
\hline Events & 0 & 0 & 0 & 0 & 0 & 0 \\
\hline Agents and Tasks in Elixir & 2 & 2 & 0 & 0 & 0 & 4 \\
\hline Synchronous Task Stream & 0 & 0 & 0 & 0 & 0 & 0 \\
\hline Pangram & 1 & 2 & 1 & 0 & 0 & 4 \\
\hline Parallel Letter Frequency & 1 & 0 & 0 & 0 & 0 & 1 \\
\hline 17-dining-philosophers & 0 & 0 & 0 & 0 & 0 & 0 \\
\hline Parallel Letter Frequency & 0 & 0 & 0 & 0 & 0 & 0 \\
\hline Elixir Sorting & 0 & 0 & 0 & 0 & 0 & 0 \\
\hline Total & 7 & 8 & 2 & 0 & 0 & 16 \\
\hline
\end{tabular}


para encontrar as falhas definidas na taxonomia de falhas. A Tabela 10 apresenta a quantidade de dados de teste presente no conjunto final de casos de teste para cada um dos programas do benchmark.

Por fim, a partir da execução dos programas defeituosos com os dados de teste, é possível encontrar as falhas nos programas, como apresentado na taxonomia de falhas. A última coluna da Tabela 10 apresenta as falhas apresentadas pelos programas defeituosos de cada um dos programas do benchmark.

Table 10: Dados de teste e falhas encontradas

\begin{tabular}{l|l|l}
\hline Programa & Dados de teste & Falhas encontradas \\
\hline Exemplo Spawn_Monitor & 4 & Finalização Precoce \\
\hline Yield_Many & 1 & Dados incorretos \\
\hline Elixir Study & 4 & $\begin{array}{l}\text { Deadlock Comporta- } \\
\text { mental, Finalização } \\
\text { Precoce }\end{array}$ \\
\hline Events & 1 & $\begin{array}{l}\text { Function Clause Erro, } \\
\text { Deadlock Comporta- } \\
\text { mental, Finalização } \\
\text { Precoce }\end{array}$ \\
\hline Agents and Tasks in Elixir & 2 & Finalização Precoce \\
\hline Synchronous Task Stream & 9 & - \\
\hline Pangram & 22 & Dados incorretos \\
\hline Parallel Letter Frequency & 1 & Dados incorretos \\
\hline 17-dining-philosophers & 1 & $\begin{array}{l}\text { Argument Error, Da- } \\
\text { dos incorretos, Dead- } \\
\text { lock Comportamental, } \\
\text { Intercalação de men- } \\
\text { sagem incorreta }\end{array}$ \\
\hline Parallel Letter Frequency & 2 & $\begin{array}{l}\text { Deadlock Comporta- } \\
\text { mental, Finalização } \\
\text { Precoce }\end{array}$ \\
\hline Elixir Sorting & & $\begin{array}{l}\text { Argument Error, Dead- } \\
\text { lock Comportamental, } \\
\text { Finalização Precoce }\end{array}$ \\
\hline & &
\end{tabular}

\section{CONCLUSÃO}

O Elixir é uma linguagem de programação que suporta o desenvolvimento de aplicações concorrentes e distribuídas de forma dinâmica e moderna. Ao desenvolver aplicações concorrentes, deve-se levar em consideração características como a comunicação, sincronização e o não determinismo intrínsecas à programação concorrente. Nesse cenário, as atividades de verificação, validação e teste precisam considerar essas características durante sua aplicação.

Como uma forma de auxiliar as atividades de verificação, validação e teste de programas concorrentes em Elixir, este artigo apresentou uma taxonomia de falhas englobando as funções presentes nos módulos Kernel e Task. A taxonomia foi dividida em dois grupos. O grupo Exceções da linguagem Elixir contém três falhas: (1) Argument Error, (2) Function Clause Error e, (3) Match Error. O grupo Falhas relacionadas à programação concorrente contém erros relacionados ao comportamento do programa e resultado gerado. Nesta categoria estão presentes: (1) Dados incorretos, (2) Deadlock comportamental, (3) Finalização precoce e (4) Intercalação de mensagem incorreta.

Por mais que existam taxonomias de defeitos para programas concorrentes, a taxonomia apresentada nesse trabalho abrange as características da linguagem de programação Elixir. A taxonomia de falhas pode ser utilizada para examinar mecanismos de detecção de falhas e servir como suporte para a definição de critérios e ferramentas de teste de programas concorrentes em Elixir.

Como trabalhos futuros, espera-se expandir a taxonomia de falhas considerando defeitos nos outros módulos do Elixir não abrangidos nesse trabalho. Além disso, com base na taxonomia de defeitos definida, será criado um conjunto de operadores de mutação para programas concorrentes em Elixir que irá embasar a aplicação do critério de teste de mutação da técnica de teste baseada em defeitos.

\section{REFERENCES}

[1] Gregory R. Andrews. Concurrent programming: principles and practice. Benjamin/Cummings Publishing Co., San Francisco, USA, 1991.

[2] Simone R. S. Souza, Paulo S. L. Souza, Silvana M. Melo, Rodolfo A. Silva, and Silvia R. Vergilio. Teste de programas concorrentes. In Marcio E. Delamaro, Mario Jino, and Jose Maldonado, editors, Introdução ao Teste de Software, volume 2, pages 261-296. Elsevier Editora Ltda, Rio de Janeiro, BR, 2016.

[3] Allan Gottlieb and George S. Almasi. Highly parallel computing. Benjamin/Cummings, Redwood City, CA, 1989.

[4] Maarten Van Steen and Andrew S. Tanenbaum. Sistemas Distribuídos: princípios e paradigmas. Prentice Hall, São Paulo, BR, 2009.

[5] Ananth Grama, Vipin Kumar, Anshul Gupta, and George Karypis. Introduction to parallel computing. Pearson Education, Essex, UK, 2003.

[6] Tiago Davi. Elixir: Do zero à concorrência. Casa do Código, São Paulo, BR, 2017.

[7] André Albuquerque and Daniel Caixinha. Mastering Elixir. Packt Publishing, Birmingham, UK, 2018.

[8] Sean Callan, Tajinder Chumber, and George Mantzouranis. A collection of companies using elixir in production. Disponível em: https://elixir-companies. com/en, 2019. Acesso em: 13 Jul. 2019.

[9] Ulisses Almeida. Learn functional programming with elixir: New Foundations for a New World. Pragmatic Bookshelf, USA, 2018.

[10] Boris Beizer. Software Testing Techniques. Van Nostrand Reinhold Co., New York, USA, 1990.

[11] A. Jefferson Offutt, Jeff Voas, and Jeff Payne. Mutation operators for Ada. Technical report, George Mason University, 1996.

[12] Jeffrey S. Vetter and Bronis R. Supinski. Dynamic software testing of MPI applications with umpire. In 2000 ACM/IEEE conference on Supercomputing, pages 1-10, Washington, DC, USA, 2000. IEEE Computer Society.

[13] Glenn R. Luecke, Hua Chen, James Coyle, Jim Hoekstra, Marina Kraeva, and Yan Zou. MPI-CHECK: a tool for checking Fortran 90 MPI programs. Concurrency and Computation: Practice and Experience, 15(2):93-100, 2003.

[14] Bettina Krammer, K. Bidmon, Matthias Stefan Müller, and Michael M. Resch. MARMOT: An MPI analysis and checking tool. In G. R. Joubert, W. E. Nagel, F. J. Peters, and W. V. Walter, editors, Parallel Computing, volume 13, pages 493-500. Elsevier, Dresden, GER, 2003.

[15] Jayant DeSouza, Bob Kuhn, Bronis R. de Supinski, Victor Samofalov, Sergey Zheltov, and Stanislav Bratanov. Automated, scalable debugging of MPI programs with intel message checker. In Workshop on Software engineering for high performance computing system applications, pages 78-82, New York, USA, 2005. ACM.

[16] Jan B. Pedersen. Classification of programming errors in parallel message passing systems. In Communicating Process Architectures, pages 363-376, Edinburgh, Sld, 2006. IOS.

[17] Shan Lu, Soyeon Park, Eunsoo Seo, and Yuanyuan Zhou. Learning from mistakes: A comprehensive study on real world concurrency bug characteristics. In 13th International Conference on Architectural Support for Programming Languages and Operating Systems, pages 329-339, New York, NY, USA, 2008. ACM.

[18] Alper Sen. Mutation operators for concurrent SystemC designs. In 10th International Workshop on Microprocessor Test and Verification, pages 27-31, Washington, DC, USA, 2009. IEEE Computer Society.

[19] Eitan Farchi, Yarden Nir, and Shmuel Ur. Concurrent bug patterns and how to test them. In Proceedings International Parallel and Distributed Processing Symposium, pages $1-7$, Nice, FR, 2003. IEEE.

[20] Carmen Torres Lopez, Stefan Marr, Elisa Gonzalez Boix, and Hanspeter Mössenböck. A Study of Concurrency Bugs and Advanced Development Support for Actor-based Programs, pages 155-185. Springer International Publishing, Cham, 2018.

[21] Matheus D. Bordignon and Rodolfo A. Silva. Benchmark de programas concorrentes em elixir para suporte à validação de critérios e ferramentas de teste. In II Simpósio de Engenharia de Software, Dois Vizinhos, PR, 2019. UTFPR. 